\title{
Energetic particle precipitation in the Brazilian geomagnetic anomaly during the "Bastille Day storm" of July 2000
}

\author{
Masanori Nishino $^{1 *}$, Kazuo Makita ${ }^{2}$, Kiyofumi Yumoto ${ }^{3}$, Yoshizumi Miyoshi ${ }^{1}$, Nelson J. Schuch ${ }^{4}$, and Mangalathayil A. Abdu ${ }^{5}$ \\ ${ }^{1}$ Solar-Terrestrial Environment Laboratory, Nagoya University, Toyokawa 442-8507, Japan \\ ${ }^{2}$ Basic Education Series, Physics, Takushoku University, Tokyo 193-8585, Japan \\ ${ }^{3}$ Space Environment Research Center, Kyushu University, Fukuoka 812-8581, Japan \\ ${ }^{4}$ INPE, Southern Space Research Center, Rio Grande de Sul 97119-900, Brazil \\ ${ }^{5}$ INPE, Sao Jose dos Campos, Sao Paulo 12201-970, Brazil
}

(Received December 9, 2004; Revised December 1, 2005; Accepted December 2, 2005; Online published May 12, 2006)

Ionospheric absorption associated with a great geomagnetic storm on July 15-16, 2000 (the "Bastille Day storm") was observed in the Brazilian geomagnetic anomaly using a two-dimensional $4 \times 4$ imaging riometer (IRIS). In the afternoon of July 15 , weak absorption $(\sim 0.2 \mathrm{~dB})$ was observed during the initial phase of the storm; large spatial-scale absorption exceeded the IRIS field of view $(330 \times 330 \mathrm{~km})$. During the sharp magnetic decrease in the main phase of the storm, absorption was intensified $(<0.5 \mathrm{~dB})$ in the evening, showing a sheet structure with $\sim 150 \mathrm{~km}$ latitudinal width and $>330 \mathrm{~km}$ longitudinal elongation. Subsequently, absorption was intensified $(\sim 1$ $\mathrm{dB})$, having a small spatial-scale $(\sim 150 \mathrm{~km})$ in the background sheet structure and a pronounced westward drift $\left(\sim 570 \mathrm{~m} \mathrm{~s}^{-1}\right)$. In association with large magnetic fluctuations in the Bz component of the interplanetary magnetic field (IMF), the ground magnetic variation in the night sector showed large positive swings during the initial to main phases of the storm. With the subsequent southward turning of the IMF Bz, the ground magnetic variation in the evening sector showed rapid storm development. Particle fluxes measured by a geosynchronous satellite ( $L=\sim 6.6$ ) demonstrated large enhancements of low-energy protons (50-400 keV) and probably electrons (50$225 \mathrm{keV})$ during the storm's initial phase. Particle fluxes from the low-altitude NOAA satellite $(\sim 870 \mathrm{~km})$ indicated the invasion of low-energy particles into the region of $L<2$ during the main phase of the storm. These results indicate that low-energy particles injected into the outer radiation belt in association with frequent and strong substorm occurrences, were transported into the inner radiation belt through direct convective access by the storm-induced electric fields during the storm's development. These particles then precipitated into the ionosphere over the Brazilian geomagnetic anomaly. Notably, the most intense absorption could be dominantly caused by proton precipitation with energies of $\sim 40 \mathrm{keV}$.

Key words: Bastille Day storm, Brazilian geomagnetic anomaly, energetic particle precipitation, imaging riometer.

\section{Introduction}

Energetic particles in the inner magnetosphere drift longitudinally in the equatorial plane, bouncing between mirror heights in the northern and southern hemispheric ionospheres as trapped particles. The mirror heights over the South Atlantic Magnetic Anomaly (SAMA), which has the global minimum intensity of the geomagnetic field, fall to $\sim 100 \mathrm{~km}$ in altitude; in comparison, mirror heights are at $\sim 600 \mathrm{~km}$ in the magnetic conjugate of the Northern Hemisphere (Gledhill, 1976). Thus, drifting energetic particles deeply precipitate into the SAMA ionosphere and lose energy through ionization processes.

In an early study, Abdu et al. (1973) used a groundbased $30-\mathrm{MHz}$ riometer to observe unusual ionospheric absorption caused by energetic electron precipitation into the SAMA during a sudden-commencement (SC) magnetic

\footnotetext{
${ }^{*}$ Now retired from Nagoya University.
}

Copyright (c) The Society of Geomagnetism and Earth, Planetary and Space Sciences (SGEPSS); The Seismological Society of Japan; The Volcanological Society of Japan; The Geodetic Society of Japan; The Japanese Society for Planetary Sciences; TERRAPUB. storm. Gough and Abdu (1975) measured phase changes in wave propagation passing through the SAMA from the NWC transmitter $(22.3 \mathrm{kHz})$, but found no evidence of enhanced D-region ionization in the SAMA. Batista and Abdu (1977) and Abdu et al. (1981) compared very low frequency (VLF) phase perturbations that propagated within the SAMA with simultaneous ionospheric Es layer parameters; those studies revealed that after the onset of magnetic disturbance, there was some delay in the occurrence of phase perturbations due to particle precipitation.

From balloon experiments in the SAMA, Pinto and Gonzalez (1986) detected intensification in X-ray flux corresponding to an integrated energy flux of $\sim 2.7 \times 10^{-4}$ ergs $\mathrm{cm}^{-2} \mathrm{~s}^{-1}$ in the energy range of $100 \mathrm{keV}$ to $1 \mathrm{MeV}$ in association with a strong geomagnetic storm. Pint et al. (1990) derived the logarithmic relationship between the amplitudes of VLF $(13.6 \mathrm{kHz})$ phase perturbations and electron fluxes predicted from simultaneous balloon X-ray measurements during seven moderate to large geomagnetic storms. From a stratospheric balloon experiment, Jayanthi et al. (1997) detected intensification of electron precipita- 
tions in the SAMA during mild geomagnetic storms associated with two successive southward turnings of the interplanetary magnetic field (IMF) Bz component. The derived electron spectrum showed a steep increase at energies below $200 \mathrm{keV}$.

From satellite-based particle observations, Pinto and Gonzalez (1989) reviewed energetic electron precipitation events in the SAMA and presented various physical mechanisms responsible for the electron precipitation. Nagata et al. (1985) demonstrated maximum fluxes of electrons (0.19-3.2 MeV) and protons (0.64-35 MeV) over the SAMA using data from the OHZORA satellite (350-850 $\mathrm{km}$ altitudes).

Many additional papers have also indicated energetic particle precipitation in the SAMA. However, previous ground- and balloon-based observations of the SAMA have primarily focused on detecting the precipitation of energetic particles and estimating their energy fluxes. Satellite observations can survey a global distribution of energetic particle precipitation from multiple orbital data. To understand the dynamics of energetic particles in the radiation belt and their precipitation processes into the SAMA, continuous and long-term investigations of temporal and spatial precipitation changes from magnetically quiet to disturbed periods are required.

Badhwar (1997) reported that in June 1995, the center of the SAMA was drifting westward at $\sim 30^{\circ} \mathrm{S}, 45^{\circ} \mathrm{W}$, off the southeastern coast of Brazil, based on dose rate data from the Skylab and Mir orbital stations. The U.S./U.K. World Magnetic Chart-Epoch 2000 data set indicates that the minimum total intensity of the geomagnetic field is situated near the southeastern Brazilian coastline. Therefore, it is important to reinvestigate energetic particle precipitation in the SAMA using ground-based observations.

To investigate temporal and spatial changes in energetic particle precipitation in the SAMA, we began the Imaging Riometer for Ionospheric Study (IRIS) project in May 1999 at the Instituto Nacional de Pesquisas Espacias, Southern Space Observatory (INPE-SSO; $29.4^{\circ} \mathrm{S}, 53.8^{\circ} \mathrm{W}, L=\sim$ 1.2) near Santa Maria, Brazil. In this area, the total intensity of the geomagnetic field was 22,855 nT in 2000 (Trivedi, personal communication). The IRIS has the great advantage of being able to measure the spatial-scale, shape, and motion of ionospheric absorption. In a previous paper (Nishino et al., 2002), we described unusual night-time ionospheric absorption characterizing energetic particle precipitation in the Brazilian geomagnetic anomaly in association with the great geomagnetic storm (minimum $D s t=-164 \mathrm{nT}$ ) on September 22-23, 1999. A remarkable feature was an eastward drift motion of the unusual absorption showing a sheet structure of east-west elongation, from which we estimated precipitating electrons of $\sim 20 \mathrm{keV}$ energies (Nishino et al., 2002).

This paper presents ionospheric absorption associated with the great geomagnetic storm (minimum Dst $=-301$ nT) on July 15-16, 2000, referred to as the "Bastille Day storm". The evening absorption showed a pronounced westward drift that differed from the previous absorption drift in September 1999. Comparison of the Bastille Day storm absorption with magnetic field data and satellite-derived parti- cle data suggests that the absorption plausibly characterizes the energetic particle precipitation into the Brazilian geomagnetic anomaly. We also discuss physical mechanisms of energetic particle transport into the inner radiation belt and energetic particle precipitation into the Brazilian geomagnetic anomaly.

\section{Ionospheric Absorption during the Bastille Day Storm}

The IRIS antenna installed at the INPE-SSO is composed of a two-dimensional $(4 \times 4)$ array with half-wavelength dipoles at $38.2 \mathrm{MHz}$, producing $4 \times 4$ beams toward the ionosphere using Butler matrix phasing circuits that combine north-south and east-west arrays. The IRIS field of view (FOV) is $\sim 330 \mathrm{~km}$ in the north-south and east-west cross-sections near the zenith at an altitude of $100 \mathrm{~km}$. Cosmic radio noise $(\mathrm{CRN})$ data from the $4 \times 4$ beams were converted to $8 \times 8$ beam data by interpolation and extrapolation to match the commonly used PC analysis program for the $8 \times 8$ beam data of the Ny Ålesund IRIS at Svalbard in the Arctic. The north-to-south and east-to-west arrays were assigned reference labels of $\mathrm{N} 1, \mathrm{~N} 2, \ldots, \mathrm{N} 8$ and $\mathrm{E} 1$, $\mathrm{E} 2, \ldots, \mathrm{E} 8$, respectively (N4E4 is an example of a combined array). Ionospheric absorption was measured using the difference between quiet-day curves (QDCs) with background CRN and those with decreased CRN intensities due to ionospheric absorption. Detailed technical descriptions have been presented by Nishino et al. (1993).

Plate 1 shows time variations of the QDC (yellow curve) and CRN intensity (white curve) on July 15 for the southwesternmost beam (N6E8). The CRN intensity decreased weakly from $\sim 15: 00$ UT and attained a minimum at $\sim 21: 00$ UT, implying maximum absorption. Note that radio noise did not interfere with CRN intensity readings except during a short period just after 14:30 UT. The good quality and non-fluctuation of the QDC allowed for a highly accurate estimation of the absorption intensity. The next section describes a favorable ionospheric condition bearing no interfering radio noise, using the ionospheric parameters measured by an ionosonde in Brazil.

Figure 1 shows variation in hourly Dst indices during

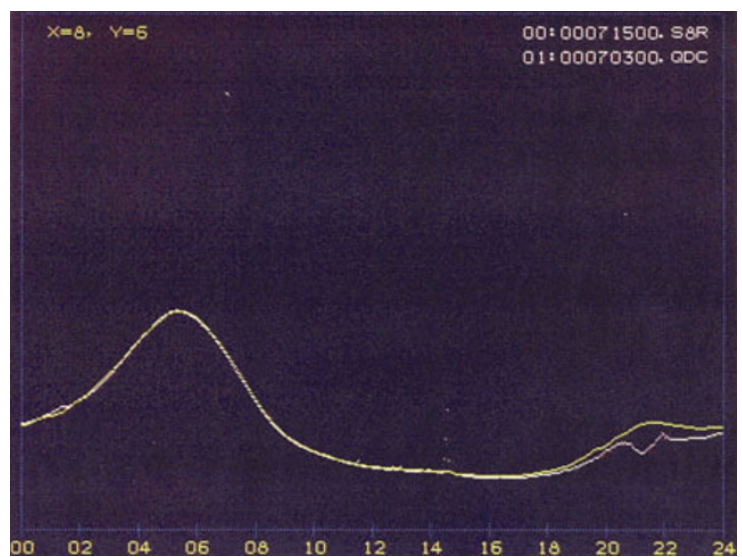

Plate 1. 24-h variations in the QDC (quiet-day curve; yellow curve) of July 2000 and cosmic radio noise intensity on July 15 (white curve) for the southwestern beam (N6E8). 


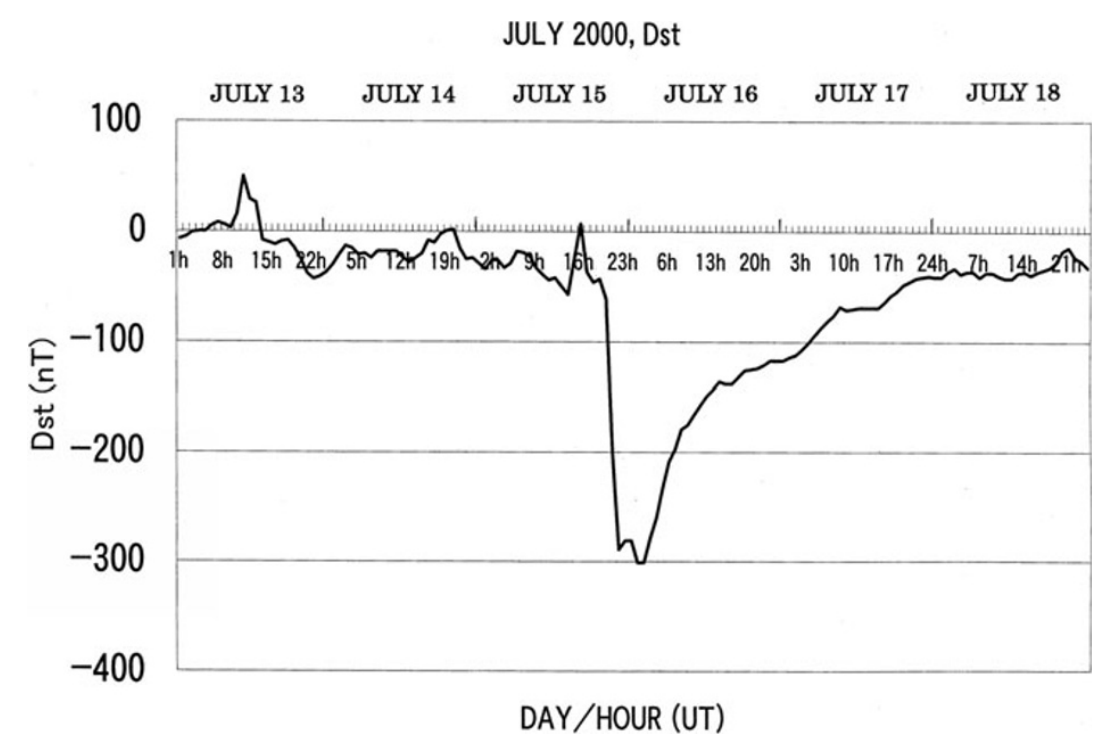

Fig. 1. Time variation of hourly Dst indices from 13 to 18 July 2000.

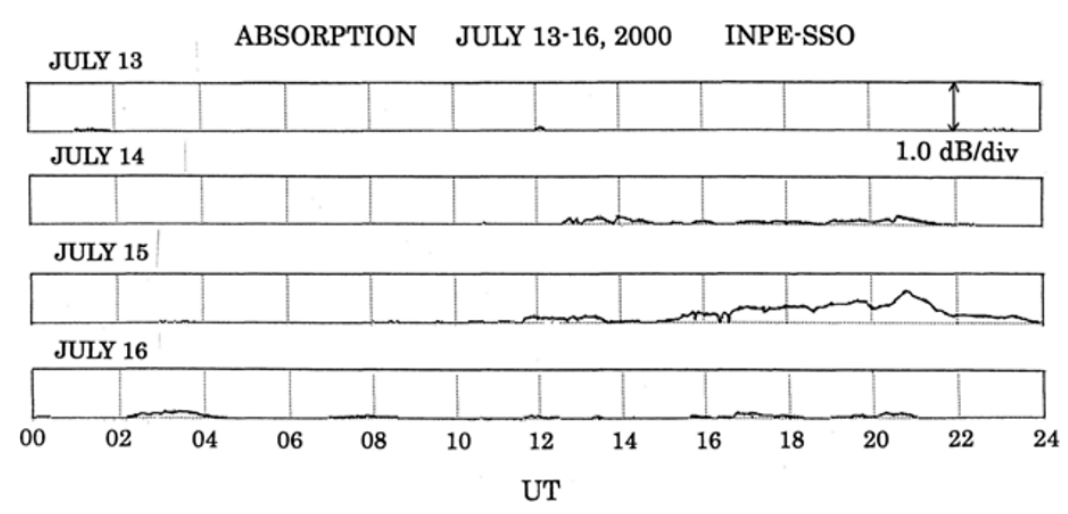

Fig. 2. Zenith beam (N4E4) 24 h variations in ionospheric absorption during July 13-16, 2000.

July 13-18, 2000. A sudden increase in Dst occurred at $\sim$ 13:00 UT on July 13, followed by slow Dst fluctuations until $\sim$ 14:00 UT on July 15 . The sudden commencement (SC) of the Bastille Day storm occurred at $\sim 15: 00$ UT on July 15; the initial phase of the storm lasted until $\sim 20: 00$ UT, when a sharp decrease in the Dst began. The main phase of the storm developed rapidly in $<3 \mathrm{~h}$, followed by a small positive swing at 23:00 UT; the minimum Dst (-301 nT) was recorded at $\sim 01: 00$ UT on July 16 . Thereafter, the Dst recovered gradually and returned to nearly $0 \mathrm{nT}$ on July 19. Thus, this variation is categorized as a great geomagnetic storm.

Figure 2 shows $24 \mathrm{~h}$ variations in ionospheric absorption on July 13-16, 2000 for the zenith beam (N4E4). Absorption intensity is plotted as averages for $128 \mathrm{~s}$ intervals, and the intensity scale is $1.0 \mathrm{~dB}$ div. $^{-1}$. Local time at Santa Maria is $3.5 \mathrm{~h}$ earlier than UT (LT $=\mathrm{UT}-3.5 \mathrm{~h})$. No absorption was observed on July 13, but weak absorption $(<0.2 \mathrm{~dB})$ was detected at $\sim 13: 00$ to $22: 00 \mathrm{UT}$ on July 14. On July 15 , weak absorption $(<0.2 \mathrm{~dB})$ occurred from $\sim 11: 30$ to $14: 30$ UT (08:00-11:00 LT). Thereafter, weak absorption was observed at $\sim 15: 00 \mathrm{UT}$; absorption slightly intensified $(\sim 0.3 \mathrm{~dB})$ at $\sim 16: 40$ UT and again intensified $(0.4-0.5 \mathrm{~dB})$ at $\sim 18: 40 \mathrm{UT}$, lasting until $\sim 20: 00$
UT. That afternoon absorption corresponds to the time period of the positive swing (initial phase) of the Dst variation (see Fig. 1). Maximum intensification $(\sim 0.7 \mathrm{~dB})$ occurred at $\sim 21: 00$ UT (17:30 LT) in the evening, corresponding to a sharp decrease (main phase) in the Dst variation. On July 16 , very weak absorption $(<0.2 \mathrm{~dB})$ was observed from $\sim 02: 00$ to 04:00 UT and 17:00 to 18:00 UT in the Dst recovery phase. In Plate 2, we display two-dimensional images of the most intense absorption at $\sim 21: 00$ UT on July 15.

Plate 2 displays a time series of absorption images from $\sim 20: 30$ to $\sim 21: 30$ UT on July 15 . The absorption intensity is represented by the color-coded bar $(0.0-1.0 \mathrm{~dB})$ below the images. In the image, north is upward (toward the Equator), and east is to the right. Each image shows a time integration of $256 \mathrm{~s}$. From 15:00 to 19:00 UT, weak $(<0.5 \mathrm{~dB})$, large-scale absorption expanded and exceeded the IRIS FOV; although absorption was contaminated at the northernmost beams by fluctuations in the QDCs caused by radio noise interference (data not shown). After disappearance of the radio noise interference, usual F-region absorption appeared at the northeast corner in the FOV until $\sim 20: 40$ UT. At $\sim 20: 45$ UT, intensified, shapeless absorption $(\sim 0.8 \mathrm{~dB})$ appeared in the north, showing a grad- 


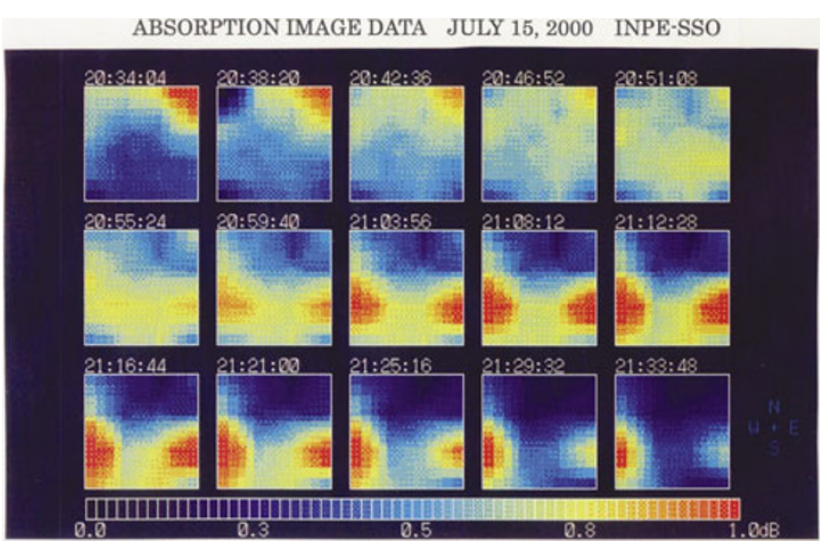

Plate 2. Time series of absorption images from $\sim 20: 30$ to $\sim 21: 30$ UT on July 15, 2000. Absorption intensities are represented by the color-coded bar below the images.

\section{ABSORPTION JULY 15, 2000 INPE-SSO}

(a) WEST

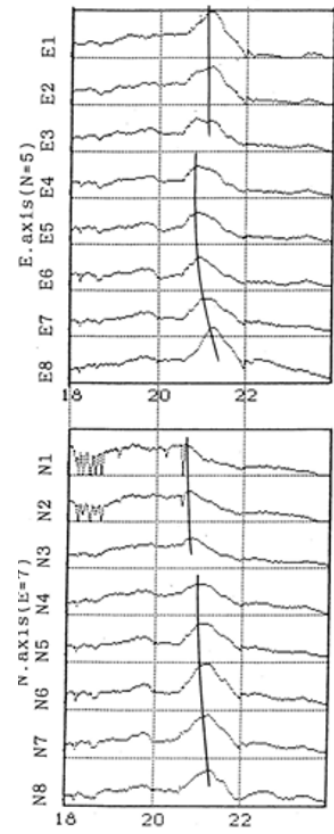

UT (b) EAST
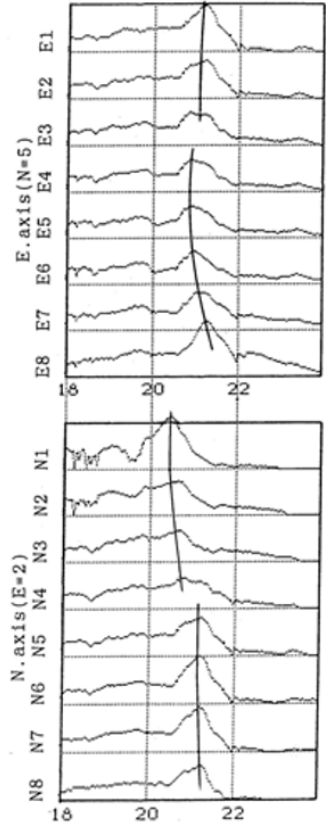

UT
Fig. 3. Time variations of the absorption intensities during 18:00-24:00 UT on July 15, 2000 for east-west beams scanned at the N5 array (upper panels) and for north-south beams scanned at the E7 (lower left) and E2 arrays (lower right).

ual southward (poleward) motion; this absorption immediately extended westward, changing to a sheet structure with $\sim 150 \mathrm{~km}$ latitudinal width and $>330 \mathrm{~km}$ east-west elongation. Thereafter, the absorption was most intensified $(\sim 1$ $\mathrm{dB}$ ) at a small spatial-scale of $\sim 150 \mathrm{~km}$ in the eastern and western parts of the sheet structure. The intensified absorption to the east weakened, followed by a subsequent weakening of absorption to the west.

Figure 3 shows time variations (18:00-24:00 UT) in the absorption intensities of the scanned beams to examine the movements of the most intense absorption at $\sim 21: 00$ UT. The upper two panels show east-west intensities (E1-E8) scanned at the N5 array. The lower left and right panels illustrate the north-south intensities (N1-N8) scanned at the E7 array (western part) and E2 array (eastern part), respectively. The upper two panels show that the absorption peaks in the western beams (E4-E8) had a pronounced westward shift, as depicted by the solid curves, indicating a westward drift velocity of $\sim 100 \mathrm{~m} \mathrm{~s}^{-1}$ at an altitude of $100 \mathrm{~km}$. This westward drift is a unique feature that has not been shown in previous studies. The absorption peaks in the eastern beams (E1-E3) also shifted westward, but this shift was not pronounced. In the lower two panels, the absorption peaks in the southern beams (N4-N8) shifted southward (poleward motion) in both the western and eastern parts. Note that the absorption peaks in the northern beams (N1-N4) in the eastern part represent usual F-region absorption, as seen in the northeast corner of the FOV in Plate 2.

Focusing on the afternoon-evening absorption on July 15 associated with the great geomagnetic storm, the absorption features are summarized as follows. Absorption with gradual intensification $(<0.5 \mathrm{~dB})$ was observed in the afternoon during the initial phase of the storm. The afternoon absorption showed large-scale expansion exceeding the IRIS-FOV. Intensified absorption $(\sim 0.8 \mathrm{~dB})$ occurred in the evening during the main phase of the storm. The evening absorption had a sheet structure with narrow latitudinal width $(\sim 150 \mathrm{~km})$ and long longitudinal elongation $(>330 \mathrm{~km})$ : The maximum intensification $(\sim 1 \mathrm{~dB})$ occurred on a small scale $(\sim 150 \mathrm{~km})$ at the western and eastern parts of the sheet structure. The maximum evening absorption showed a pronounced westward drift.

\section{Ionospheric Conditions}

For accurate measurements of ionospheric absorption, high-quality QDCs are essential. Background CRN at 38.2 $\mathrm{MHz}$ is sometimes contaminated by radio-noise interference propagated at long distances, depending on the ionospheric F-region conditions. Contaminated CRN lessens the QDC quality, resulting in low-quality ionospheric absorption measurements. In association with the September 1999 storm, the low-latitude ionospheric F-region condition showed a foF2 increase and $\mathrm{h}^{\prime} \mathrm{F} 2$ decrease in ionospheric parameters during the unusual absorption (Nishino et al., 2002).

This section also describes the low-latitude ionospheric condition during the absorption associated with the present storm. Figure 4 shows time variations of the ionospheric parameters (foEs, foF2, and $h^{\prime} \mathrm{F} 2$ ) on July 15-16, 2000, measured by the ionosonde at Cachoeira Paulista $\left(22.7^{\circ} \mathrm{S}\right.$, $\left.45.0^{\circ} \mathrm{W}\right)$. Relative foF2 increases and $h^{\prime} \mathrm{F} 2$ decreases occurred from $\sim 12: 00$ to 19:00 UT, causing QDC fluctuations at the northernmost beams due to increases in radio-noise interference and resulted in irregular variations with apparent absorption, although the QDCs near the zenith did not fluctuate, as shown in Plate 1 . Note that a large foF2 decrease to $\sim 4 \mathrm{MHz}$ (in the left vertical axis) occurred after $\sim 20: 00$ UT on July 15, indicating the absence of radionoise interference at the IRIS station due to the lack of radio reflection in the lower-latitude F-region ionosphere. The large increase in $\mathrm{h}^{\prime} \mathrm{F} 2$ between 600 and $700 \mathrm{~km}$ altitudes (the right vertical axis) after $\sim 20: 00$ UT indicates an up- 


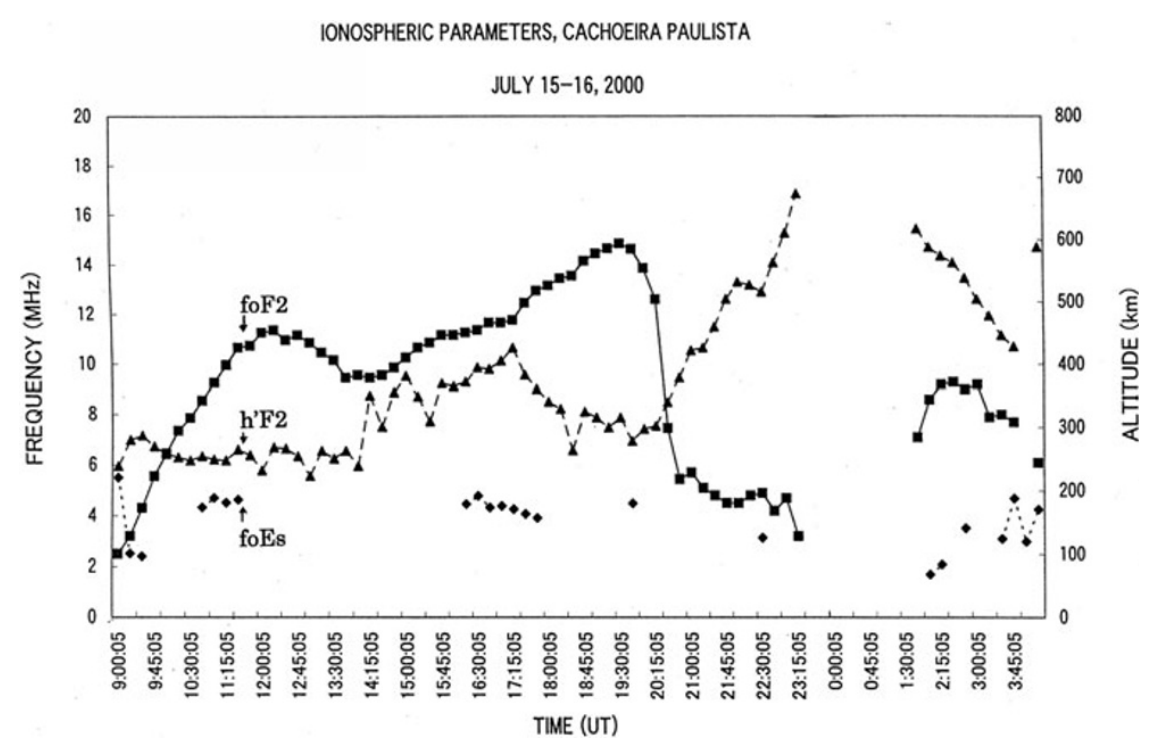

Fig. 4. Time variations in ionospheric parameters of foEs (in frequency), foF2 (in frequency), and $\mathrm{h}^{\prime} \mathrm{F} 2$ (in altitude) on July $15-16,2000$ measured by the ionosonde at Cachoeira Paulista, Brazil. Measurements were taken every 15 min.

lift of the low-latitude $\mathrm{F}$ region. The F-region uplift can be attributed to eastward electric fields in the low-latitude ionosphere penetrating from the magnetosphere in association with storm development (Tanaka, 1986). Uplift in the F-region due to storm-induced eastward electric fields in the Brazilian sector associated with the Bastille Day storm has also been reported (Basu et al., 2001).

\section{Discussion}

The above section summarized ionospheric absorption observed in the Brazilian geomagnetic anomaly, as associated with the Bastille Day storm and absorption features on July 15 . This section compares the absorption features with magnetic field and satellite-derived particle data. Comparisons indicate particle precipitation into the Brazilian geomagnetic anomaly. The physical mechanisms of energetic particle transport into the inner radiation belt and their precipitation into the anomaly are also discussed.

Figure 5 shows time variations of the IMF Bz component from WIND satellite data (upper panel) and the geomagnetic H component at Santa Maria (SMA) in the Brazilian anomaly (middle panel) and at Hermunus (lower panel, HER; geomagnetic lat., $33.7^{\circ} \mathrm{S}$ ) in South Africa on July 1516,2000 . When the WIND satellite was located 70 Re upstream the Earth, the propagation time between the WIND and the Earth ionosphere through the magnetopause was short ( 9.5 min), taking into account high-speed $(\sim 1000$ $\mathrm{km} \mathrm{s}^{-1}$ ) streams of solar wind. The IMF Bz component suddenly swung to positive at $\sim 14: 30$ UT on July 15 , followed by large magnetic fluctuations $( \pm 20-40 \mathrm{nT})$ until $\sim 19: 00$ UT and an immediate turn southward, attaining minimum intensity $(\sim-60 \mathrm{nT})$ at $\sim 20: 00 \mathrm{UT}$. Thereafter, recovery took place for several hours. The geomagnetic $\mathrm{H}$ component at Santa Maria showed rather small magnetic fluctuations responding to the large IMF fluctuations, followed by a sharp decrease to a minimum intensity $(>-200 \mathrm{nT})$, indicating the rapid $(<3 \mathrm{~h})$ and large development of storm-ring currents in the radiation belt. At Hermunus, in the night
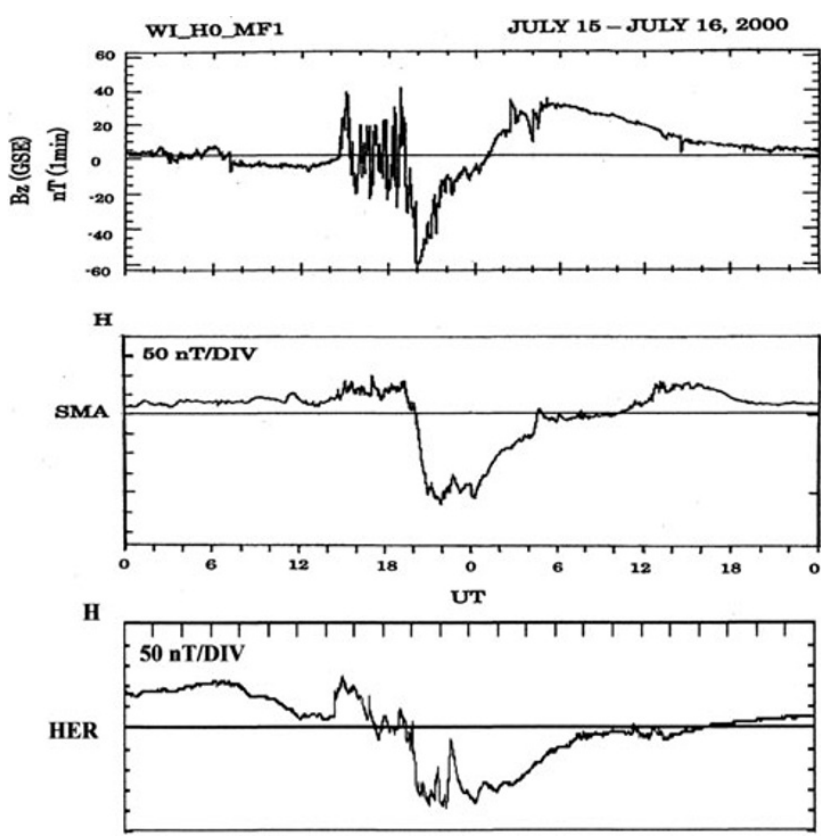

Fig. 5. Time variations in the IMF Bz component from WIND satellite data (upper panel) and the geomagnetic $\mathrm{H}$ component at Santa Maria (middle panel) and Hermunus (lower panel) on July 15-16, 2000.

sector during the storm, the geomagnetic $\mathrm{H}$ component showed large positive excursions responding to the large IMF fluctuations, indicating injections of abundant particles into the radiation belt. Associated with the IMF fluctuations, geomagnetic $\mathrm{H}$ components at the IMAGE magnetometer network stations in high latitudes (Lühr et al., 1998) showed multiple magnetic swings with large positive amplitudes (1000-2000 nT; data not shown), indicating strong and frequent substorm occurrences from $\sim 15: 00$ to 19:00 UT. Strong substorm occurrences were also recognized by increased Kp indices in the storm's initial phase, as shown in Plate 3. These findings suggest that an abun- 


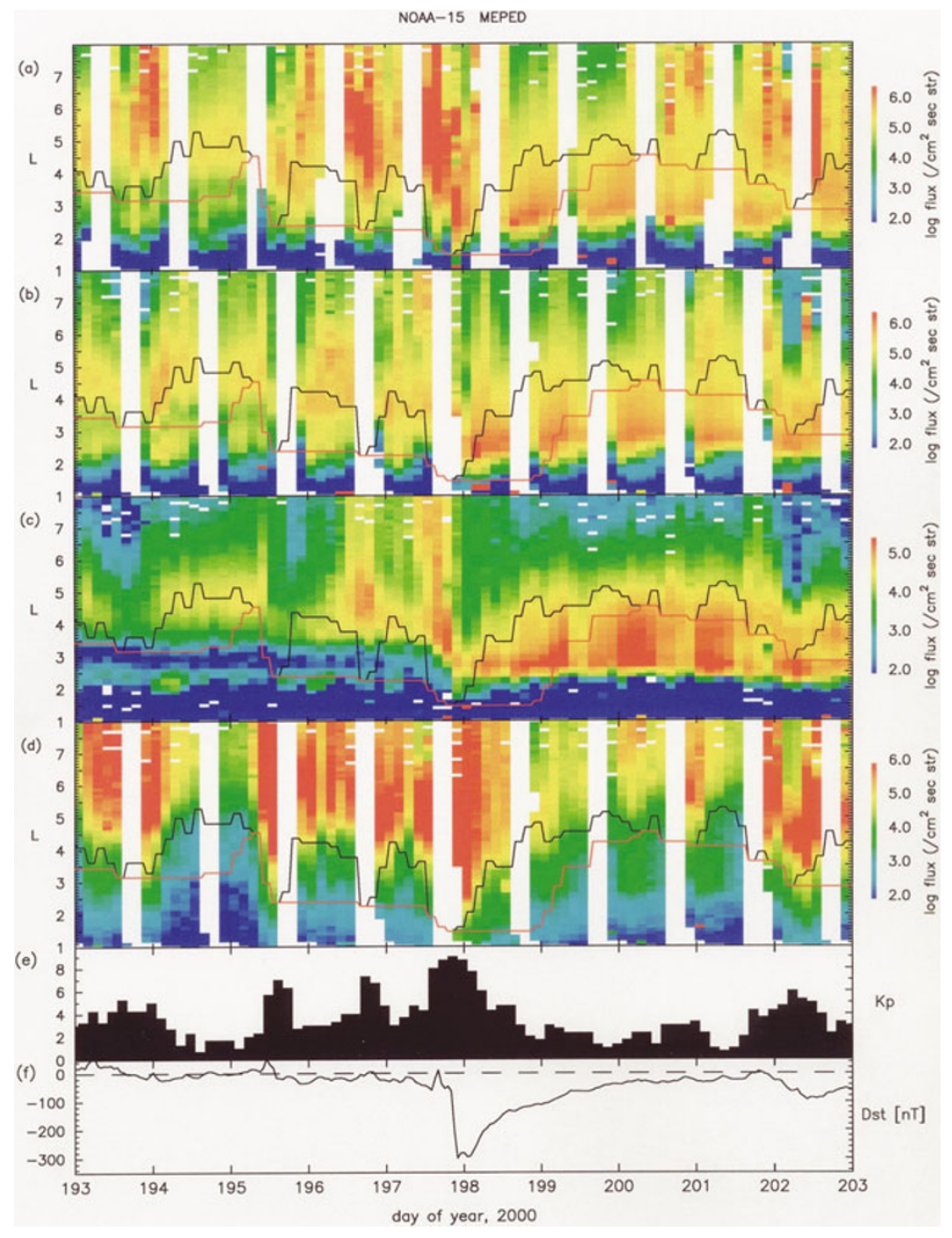

Plate 3. Time variations in particle log-fluxes with L values for days of the year 193-203 days in 2000 from MEPED data obtained by the low-altitude NOAA-15 satellite. The following L-t diagrams are displayed: (a) $30 \mathrm{keV}$ electrons in the morning sector, (b) $30 \mathrm{keV}$ electrons in the evening sector, (c) $300 \mathrm{keV}$ electrons in the morning sector, and (d) 30 to $80 \mathrm{keV}$ protons in the evening sector. Log-flux values are displayed by color-coded bars on the right-hand side. Time variations in Kp and Dst indices are displayed in panels (e) and (f), respectively.

dance of substorm energetic particles could have been injected frequently into the outer radiation belt during the initial phase of the storm, resulting in a large development of storm-ring currents. As described below, simultaneous particle flux data from a geosynchronous satellite provide direct evidence of energetic particle injections.

Figure 6 shows time variations of energetic electron (upper two panels) and proton (lower two panels) fluxes on July 15 observed by the particle analyzer onboard the Los Alamos National Laboratory (LANL) satellite $(L=\sim 6.6)$. Fluxes are distinguished in the low- and high-energy bands. Electron data in the low-energy band (50-225 keV) are lacking until $\sim$ 19:00 UT, but showed increases between $\sim$ 19:00 and $\sim 20: 00$ UT. Electron fluxes in the highenergy band (315-1500 keV) increased slightly at $\sim 11: 00$ UT; large increases followed at $\sim 14: 45$ UT, lasting $1-$ $2 \mathrm{~h}$. In contrast, proton fluxes in the low-energy band (50$400 \mathrm{keV}$ ) showed slight increases at $\sim 8: 30 \mathrm{UT}$, large increases from $\sim 10: 30$ to $\sim 12: 00 \mathrm{UT}$, and small increases from $\sim 12: 00$ to $\sim 14: 45$ UT. Large increases in the low- energy protons occurred from $\sim 14: 45$ to $\sim 19$ :00 UT. Variation in proton fluxes in the high-energy band (1.2-5.0 $\mathrm{MeV}$ ) was similar to that of the low-energy protons, but the fluxes were small. The above variations indicate large increases of protons and electrons at $\sim 14: 45$ UT in association with the initial IMF impulse at $\sim 14: 30 \mathrm{UT}$; however, only low-energy protons and probably low-energy electrons sustained large flux increases until $\sim 19: 00$ UT. These increases ended $\sim 3 \mathrm{~h}$ earlier $(\sim 19: 00$ UT) than the absorption ended $(\sim 22: 00 \mathrm{UT})$. This time difference may reflect transportation from the satellite $(L=\sim 6.6)$ to the low-L regions and longitudinal drift. Consequently, absorption from $\sim 16: 40$ to $22: 00$ UT on July 15 could have been caused by low-energy protons and probably low-energy electrons injected into the radiation belt; thus, this absorption is considered unusual absorption associated with the great magnetic storm.

On July 14 , one day before of the storm, no data were gathered on low-energy electron fluxes, and high-energy electron fluxes showed very small increases. The low- 
L9_K0_SPA LANL Particle Analyzer JULY 15, 2000

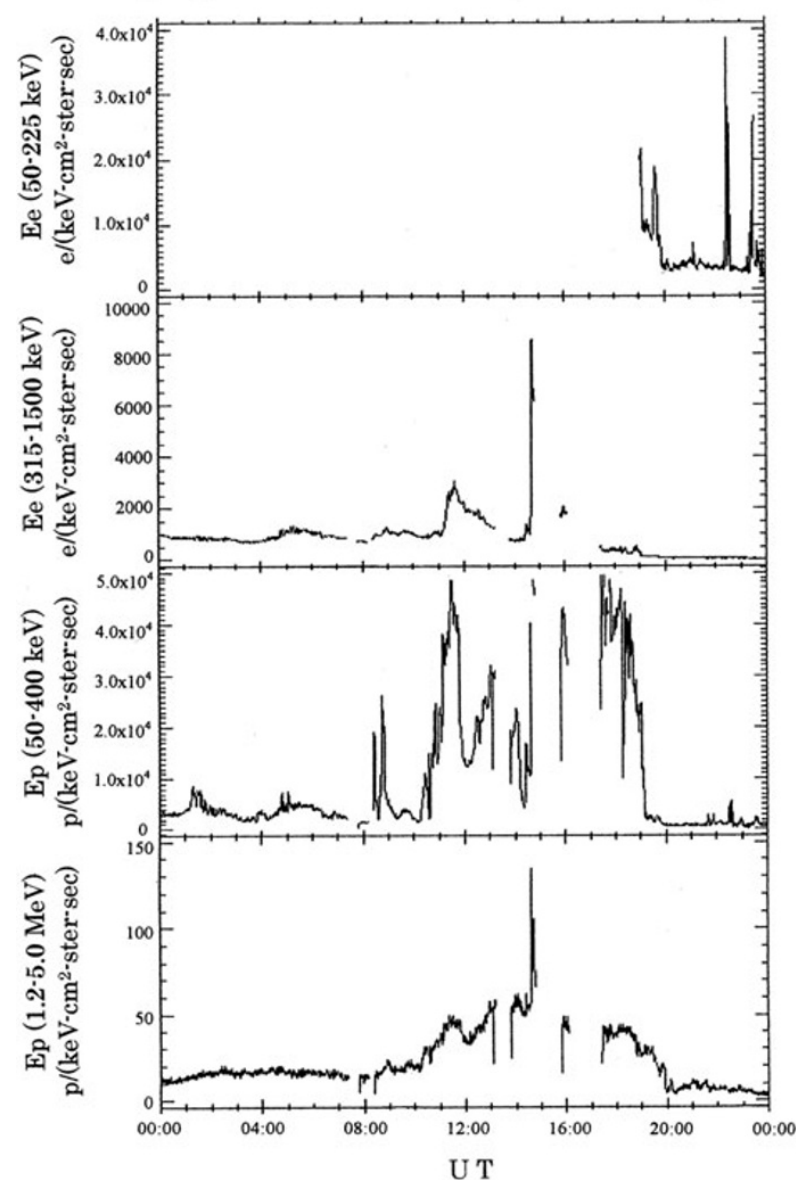

Fig. 6. Time variations in energy fluxes of electrons (upper two) and protons (lower two) on July 15, 2000 observed by the particle detector onboard the LANL satellite. Energy fluxes were distinguished in the low- and high-energy bands for electrons and protons.

and high-energy proton fluxes also showed small increases (data not shown). In addition, the time periods of the flux increases were not correlated with the absorption time ( 13:00-15:00 UT and 19:00-22:00 UT) on July 14. On July 16 during the storm's recovery phase, no flux increases of electrons and protons were seen all day. Thus, the weak absorption on July 14 and 16 was unrelated to the small increases of particle fluxes and identified as usual F-region absorption (Abdu et al., 1967).

Next, we examine particle data observed by a lowaltitude satellite as evidence of the transportation of energetic particles into low L-regions. Plate 3 shows the L-t diagram of Medium Energy Proton and Electron Detector (MEPED) data from the NOAA-15 satellite $(\sim 870 \mathrm{~km}$ altitude) in the Southern Hemisphere. One orbital period of the satellite equaled $102 \mathrm{~min}$. Integral fluxes of particles every $3 \mathrm{~h}$ are displayed by color codes. In the low-L region orbits, the $90^{\circ}$ MEPED sensor detected trapped particles at $80-120^{\circ}$ pitch angles. The upper four panels display time variations of particle fluxes with $\mathrm{L}$ values during days of the year 193-203 in 2000: (a) $30 \mathrm{keV}$ electrons in the morning sector, (b) $30 \mathrm{keV}$ electrons in the evening sector, (c) 300 $\mathrm{keV}$ electrons in the morning sector, and (d) 30 to $80 \mathrm{keV}$ protons in the evening sector (Raben et al., 1995). In the bottom two panels, time variations in (e) Kp indices and (f) Dst indices are also displayed. The particle data on July 15-16 (days 197-198) shown in panel (a) indicate that 30 $\mathrm{keV}$ electrons invaded the low-L region $(\sim 2-3)$ from the high-L $(\sim 6-7)$ region in the morning sector during the initial phase of the storm, with large integral fluxes of $10^{5}-10^{6}$ $\left(/ \mathrm{cm}^{2} \mathrm{~s} \mathrm{str}\right)$; these electrons further invaded to the lower $L$ region $(<2)$ during the main phase of the storm. Although $30 \mathrm{keV}$ electron data in the evening sector (b) were lacking during the initial to main phases of the storm, $30 \mathrm{keV}$ electrons probably also invaded the lower $L$ region $(<2)$ in the evening sector. As shown in panel (c), $300 \mathrm{keV}$ electrons invaded to $L=\sim 2$ in the morning sector, but fluxes were relatively small. The $300 \mathrm{keV}$ electrons remained near $L=\sim 2.5$ in the storm recovery phase. Panel (d) indicates that the 30 to $80 \mathrm{keV}$ protons invaded the low $L=\sim 2$ region in the evening sector near the minimum Dst and had large fluxes in the order of $10^{6}\left(/ \mathrm{cm}^{2} \mathrm{~s} \mathrm{str}\right)$. These data show that both lower-energy protons and electrons were transported into the inner radiation belt during the storm's development. The transport of low-energy particles into the region of $L=\sim 2$, as associated with great geomagnetic storms, was clarified by the radial profiles of the total ion energy density (30-300 keV/e) from AMPTE/CCE satellite data (Chen et al., 1997).

Below, we estimate approximate particle energies to discuss transportation mechanisms of the energetic particles in the inner magnetosphere. Particle energies were estimated from the longitudinal drift of the intense absorption. A quarter period $T(s)$ of longitudinal drift encircling the Earth on the equatorial plane is given for low-energy particles as follows (Fälthammer, 1973):

$$
T(s)=\frac{0.7 \times 10^{9}}{0.7+0.3 \times \sin \alpha_{e}} \frac{1}{L} \frac{1}{W},
$$

where $\alpha_{e}=45^{\circ}$ is a typical value of the equatorial pitch angles of drifting particles near the Brazilian sector (Datlowe and Imhof, 1993), $L$ is the value of the equatorial crossing of particles in distances of Earth radii, and $W$ is energy in $\mathrm{eV}$. From the east-west shift of the absorption peaks at $\sim 21: 00$ UT (see Fig. 3), we obtained a westward motion of $\sim 100 \mathrm{~m} \mathrm{~s}^{-1}$ velocity, assuming an ionospheric altitude of $100 \mathrm{~km}$. Taking into account the eastward corotation (15 $\operatorname{deg} \mathrm{h}^{-1}$ ) of the Earth's ionosphere, the net velocity of drifting particles was corrected to $570 \mathrm{~m} \mathrm{~s}^{-1}$ westward, and the quarter period of particle drift was calculated as $L=1.2$. Thus, using Eq.(1), we could estimate drifting particles with energies of $35.9 \mathrm{keV}$.

Abdu et al. (1973) revealed that the longitudinal drift of precipitating particles is subject to an $\mathbf{E} \times \mathbf{B}$ drift in addition to drift due to the magnetic field gradient in the plasmasphere. They estimated precipitating electron energies $(\sim 30$ $\mathrm{keV}$ ) from the eastward drift of the absorption associated with a great SC geomagnetic storm, assuming electric fields in the equatorial plane. Likewise, Nishino et al. (2002) estimated precipitating electron energies $(\sim 20 \mathrm{keV})$ from the eastward drift of absorption, assuming electric fields of 1.8 $\mathrm{mV} \mathrm{m}^{-1}$. For the present estimation, we neglected the effect of the electric field. Even when the electric field in the equatorial plane was assumed to be $1 \mathrm{mV} \mathrm{m}^{-1}$, deviations 
from the estimated energies $(35.9 \mathrm{keV}$ ) were $\sim 10 \%$ (Abdu et al., 1973). We consequently obtained important evidence that the most intense absorption showing westward motion may have been predominantly caused by proton precipitation with energies of $\sim 40 \mathrm{keV}$.

Lyons and Williams (1980) found that fluxes of trapped particles at $L \leq 4$ during great geomagnetic storms increased by a 1-3 Re inward radial displacement of the existing trapped particle distribution by enhanced storm-time electric fields. This proposed source for the storm-time ring current particles at $L \leq 4$ requires only the acceleration of the previously existing trapped particle population via inward displacement under conservation of the first two adiabatic invariants. However, Lyons and Schulz (1989) noted that if high-energy $(\geq 40 \mathrm{keV})$ ring-current particles have drift periods that are typically shorter than the development time of the storm, direct radial displacement could be restricted. In the equatorial plane at $L=1.2$, the first adiabatic invariant for protons was low $\left(\sim 0.18 \mathrm{MeV} \mathrm{G}^{-1}\right)$ at $B=0.22 \mathrm{G}$ (Paulikas, 1975). By the above equation, protons $(35.9 \mathrm{keV})$ had encircling drift periods of $19.8 \mathrm{~h}$, which were evidently longer than the storm development time $(\sim 3 \mathrm{~h})$. Thus, according to the theoretical calculations by Lyons and Schulz (1989), it is expected that energetic protons could have been transported to the inner radiation belt through direct convection access due to storm-induced electric fields. Likewise, it is expected that energetic electrons with energies of several tens of $\mathrm{keV}$ could be transported into the inner radiation belt (see Plate 3 ).

Finally, we discuss the physical processes of energetic particle precipitation in the Brazilian geomagnetic anomaly based on the spatial-scale of the precipitation obtained by the IRIS observations. Kohno et al. (1990) demonstrated global distributions of electron $(0.19-3.2 \mathrm{MeV})$ and proton (0.64-35 MeV) fluxes in the SAMA by statistically analyzing data from $\sim 3$ years of OHZORA satellite observations. The satellite-observed trapped particles had nearly $90^{\circ}$ pitch angles. The distributions were not selected in relation to geomagnetic activities. Strong particle enhancements $\left(>10^{5}\right.$ particles $/ \mathrm{cm}^{2} \mathrm{~s}$ str) were distributed across a broad area in $30^{\circ}$ latitudinal and $50^{\circ}$ longitudinal widths centered off the southeast coast of Brazil at 700 to $850 \mathrm{~km}$ altitudes. Greenspan et al. (1999) surveyed a global map of energetic ion count rates ( $\geq 730 \mathrm{keV}$ energies for protons) during June 1996 from the low-altitude Solar, Anomalous, and Magnetospheric Explorer (SAMPEX) satellite; that survey demonstrated a broader enhancement area with $40^{\circ}$ latitudinal and $90^{\circ}$ longitudinal widths. In contrast, the proton precipitation area in Plate 2 shows the small spatial-scale of the sheet structure, although the electron precipitation area is ambiguous because of its expansion beyond the IRIS FOV.

Torr et al. (1975) calculated relative precipitated fluxes in the SAMA using a simple model of charged-particle motion in the closed magnetic-field line, assuming a continuous injection of energetic electrons with isotropic angular distributions into the radiation belt. They obtained the longitudinal variation in the median values of precipitated electron fluxes in the Southern Hemisphere and showed a peak energy influx of $0.035 \mathrm{erg} \mathrm{cm}^{-2} \mathrm{~s}^{-1}$ near $10^{\circ} \mathrm{W}$ longitude in the anomaly at $L=2$, which corresponds to $\sim 20$
Rayleigh of the $3914 \AA$ airglow, assuming an average energy of $50 \mathrm{keV}$ per electron. The longitudinal variation was asymmetric, having a gradual slope on the western side of the peak and a sharp cutoff edge on the eastern side. Torr et al. (1975) proposed that the asymmetry was created by a buildup of stored electrons on the west side of the anomaly as electrons drifted eastward and by a sharp drop of precipitated electron flux near the center of the anomaly. Torr et al. (1975) also showed a peak enhancement of precipitated fluxes for $280 \mathrm{keV}$ protons, although the asymmetric variation was opposite to that for the electrons.

Pinto and Gonzalez (1989) reviewed the following scattering processes by wave-particle interactions in the Earth's radiation belt as mechanisms of energetic electron precipitation in the SAMA: magnetospheric wave-particle interactions, lightning or artificially induced wave-particle interactions, drift-resonance interactions, and wave-particle interactions generated by plasma instabilities. They proposed that electron precipitation results from the combination of various mechanisms. Abel and Thorne (1999) numerically simulated electron pitch angle scattering in an asymmetric magnetic field environment in the inner radiation belt. They assumed that pitch angle diffusions of $500 \mathrm{keV}$ electrons are caused by pitch-angle scattering angles of $0.01^{\circ}$ by Coulomb scattering and wave-particle interactions with plasmaspheric hiss and other VLF waves in the inner magnetosphere. Their results showed a peak in local electron precipitation flux with an asymmetric longitudinal range $\left( \pm \sim 15^{\circ}\right)$ near $45^{\circ} \mathrm{W}$ longitude in the SAMA at $L=1.35$; furthermore, the atmospheric ion production rate due to energetic electron precipitation was higher compared to that from cosmic rays and $\mathrm{H}$ Lyman $\alpha$ radiation in the 70-75 $\mathrm{km}$ altitude range at night. However, they did not simulate proton precipitation.

The weak afternoon absorption observed until $\sim 20: 00$ UT was considered the drizzle of eastward-drifting electrons and westward-drifting protons; the spatial extension of the absorption was ambiguous, as described above. Therefore, we cannot suggest definite physical mechanisms of the precipitation for the afternoon absorption. In contrast, evening absorption with small-scale intensification showed a westward drift and disappeared in the anomaly region. This absorption behavior is quite similar to the longitudinal variation of proton precipitation obtained by Torr et al. (1975). However, based on this simple model, it is unclear whether proton precipitation produced ionospheric absorption, indicating the need to examine wave-particle interactions.

Recently, from measurements of $155 \mathrm{keV}$ protons taken by the Polar satellite, Walt and Voss (2004) found intense proton fluxes during the main and early storm recovery phases at $L>4$, where ring-current models predicted that electromagnetic ion cyclotron (EMIC) waves would be generated by ring-current ions. This satellite result may suggest that EMIC wave-particle interactions cause pitchangle scattering of protons and precipitation in the Brazilian anomaly.

Gonzalez et al. (1987) proposed a quite different physical mechanism, suggesting that the distortion effect in a fair-weather electric field at balloon altitudes could be at- 
tributed to local intensification of energetic electron precipitation. They suggested the existence of fairly large ionization enhancements in the middle atmosphere during magnetically disturbed periods and considered that precipitation enhancement could occur in a fairly narrow region in the order of a few hundred kilometers in the SAMA.

Besides the above physical mechanisms, the small-scale precipitation may be due to the difference between the instrumental thresholds of the particle detector onboard the satellite and the IRIS; the IRIS may detect only strong parts in the broad precipitation area observed by the satellite.

Consequently, we cannot propose definite physical mechanisms for the particle precipitation in the Brazilian geomagnetic anomaly. We need additional data, including information on wave phenomena and atmospheric electricity in the Brazilian geomagnetic anomaly. We also require more exact information regarding the spatial extension and movement of ionospheric absorption; such information can be obtained by network observations using multiple IRISs.

\section{Conclusions}

Ionospheric absorption was observed in association with the Bastille Day storm on July 15-16, 2000 by the IRIS in the Brazilian geomagnetic anomaly. On July 15, weak absorption was observed in the afternoon during the initial phase of the storm; this large-scale absorption exceeded the IRIS FOV. Absorption was intensified in the evening during the storm's main phase, showing a sheet structure with $\sim 150 \mathrm{~km}$ latitudinal width and $>350 \mathrm{~km}$ longitudinal elongation. Subsequently, the most intense absorption occurred on a small spatial-scale in the background sheet structure and showed a pronounced westward drift. The IMF Bz component from the WIND satellite initially had large magnetic fluctuations and subsequently turned southward with large amplitudes. The ground magnetic $\mathrm{H}$ component in the night sector had large fluctuations in the initial phase of the storm. The geomagnetic $\mathrm{H}$ component in the evening sector showed the rapid development of the main phase of the storm. Particle fluxes observed by the LANL satellite ( $L=\sim 6.6$ ) demonstrated large enhancement of lowenergy particles during absorption. Particle fluxes observed by the low-altitude NOAA satellite $(\sim 870 \mathrm{~km})$ showed the invasion of large low-energy particle fluxes into the inner radiation belt during the storm's development. These results indicate that low-energy particles injected into the outer radiation belt that are associated with strong and frequent substorm occurrences are transported into the inner radiation belt through direct convective access by storm-induced electric fields and thus precipitate into the ionosphere in the Brazilian geomagnetic anomaly. In particular, the results provided important evidence that proton precipitation at energies of $\sim 40 \mathrm{keV}$ may predominantly have caused the intense absorption. For the small-scale absorption caused by proton precipitation, we speculate that EMIC wave-particle interactions may operate in the radiation belt.

Due to the small IRIS FOV, we could not determine the spatial scale and movement of the afternoon absorption exceeding the FOV. To overcome this issue, we recently installed two IRIS stations at Concepcíon $\left(37.5^{\circ} \mathrm{S}, 72.7^{\circ} \mathrm{W}\right.$, $L=\sim 1.3$ ) to the west of Santa Maria and Punta Arenas $\left(53.1^{\circ} \mathrm{S}, 71.0^{\circ} \mathrm{W}, L=\sim 1.6\right)$, at the higher-latitude side of Santa Maria, in Chile. We are presently collecting data at these two IRISs and the original Santa Maria IRIS in collaboration with the INPE (Brazil) and Universidad de Magallanes and Universidad de Concepción (Chile).

Acknowledgments. Research on the Brazilian geomagnetic anomaly reported in this paper was conducted in collaboration with the Instituto Nacional de Pesquisas Espacias (INPE) in Brazil. The IRIS observations were partly supported by the Monbu-Kagaku-Syo International Scientific Research Program. K.M. was supported by funds from the Science and Engineering Institute, Takushoku University. We thank the students at the Laboratorio de Ciencias Espiciais de Santa Maria University for maintaining the continuous IRIS observations.

The Dst indices and geomagnetic data were provided by the WDC-C2 for Geomagnetism, Kyoto University. We acknowledge Ron Repping, NASA Goddard Flight Center, for the use of WIND MFI data and E. Dors at LANL and CDAWeb for use of the particle data. IMAGE geomagnetic data were provided through the IMAGE Web.

\section{References}

Abdu, M. A., S. S. Degaonkar, and K. R. Ramanathan, Attenuation of galactic radio noise at $25 \mathrm{Mhz}$ and $21.3 \mathrm{Mhz}$ in the ionosphere over Ahmedabad during 1957-1964, J. Geophys. Res., 72(5), 1547-1554, 1967.

Abdu, M. A., S. Ananthakrishnan, E. F. Coutinho, B. A. Krishnan, and E. M. Da S. Reis, Azimuthal drift and precipitation of electrons into the South Atlantic geomagnetic anomaly during an SC magnetic storm, $J$. Geophys. Res., 78(25), 5830-5836, 1973.

Abdu, M. A., I. S. Batista, R. Piazza, and O. Massambani, Magnetic storm associated enhanced particle precipitation in the South Atlantic anomaly: Evidence from VLF phase measurements, J. Geophys. Res., 86(A9), 7533-7542, 1981.

Abel, B. and R. M. Thorne, Modeling energetic electron precipitation near the South Atlantic anomaly, J. Geophys. Res., 104(A4), 7037-7044, 1999.

Badhwar, G. D., Drift rate of the South Atlantic anomaly, J. Geophys. Res., 102, 2343-2349, 1997.

Basu, S., Su Basu, K. M. Gloves, H.-C. Yeh, S.-Y. Su, R. J. Rich, P. J. Sultan, and M. J. Keskinen, Response of the equatorial ionosphere in the South Atlantic region to the great magnetic storm of July 15, 2000, Geophys. Res. Lett., 28(18), 3577-3580, 2001.

Batista, L. S. and M. A. Abdu, Magnetic storm associated with delayed sporadic E enhancements in the Brazilian geomagnetic anomaly, J. Geophys. Res., 82(29), 4777-4783, 1977.

Chen, M., M. Schulz, and L. R. Lyons, Modeling of ring current formation and decay: A review, Magnetic Storms, Geophysical Monograph, 98, pp. 173-186, AGU, Washington, D.C., 1997.

Datlowe, D. W. and W. L. Imhof, Seasonal variations of energetic electron precipitation by cyclotron resonance with VLF waves from a groundbased transmitter, Radio Science, 28, 705-714, 1993.

Fälthammer, C. G., Motion of charged particles in the magnetosphere, in Cosmic Geophysics, edited by A. Egeland et al., p. 121, Universitiesforlaget, Oslo, 1973.

Gledhill, J. A., Aeronomic effects of the South Atlantic anomaly, Rev. Geophys. Space Phys., 14(2), 173-187, 1976.

Gonzalez, W. D., S. L. G. Dutra, and O. Pinto, Jr., Middle atmospheric electrodynamic modification by particle precipitation at the South Atlantic Magnetic Anomaly, J. Atmos. Terr. Phys., 49(4), 377-383, 1987.

Gough, M. P. and M. A. Abdu, Particle precipitation in the South Atlantic anomaly deduced from VLF propagation path measurements, J. Atmos. Terr. Phys., 37, 1379-1383, 1975.

Greenspan, M. E., G. M. Mason, and J. E. Mazur, Low-altitude equatorial ions: A new look with SAMPEX, J. Geophys. Res., 104(A9), 19,911$19,922,1999$.

Jayanthi, U. B., M. G. Pereira, I. M. Martin, Y. Stozkov, F. D’Amico, and T. Villela, Electron precipitation associated with geomagnetic activity: Balloon observation of X ray flux in South Atlantic anomaly, J. Geophys. Res., 102(A11), 24,069-24,073, 1997.

Kohno, T., K. Munakata, K. Nagata, H. Murakami, A. Nakamoto, N. Hasebe, J. Kikuchi, and T. Doke, Intensity maps of MeV electrons and 
protons below the radiation belt, Planet. Space Sci., 38(4), 483-490, 1990.

Lühr, H., A. Aylward, S. C. Bucher, A. Pajunpaa, K. Pajunpaa, T. Holmboe, and S.M. Zalewski, Westward moving dynamic substorm features observed with IMAGE magnetometer network and other ground-based instruments, Ann. Geophys., 16, 425-440, 1998.

Lyons, L. R. and M. Schulz, Access of energetic particles to storm time ring current through enhanced radial "diffusion", J. Geophys. Res., 94(A5), 5491-5496, 1989.

Lyons, L. R. and D. J. Williams, A source for the geomagnetic storm main phase ring current, J. Geophys. Res., 85(A2), 523-530, 1980.

Nagata, K., T. Khono, H. Murakami, A. Nakamoto, N. Hasebe, T. Takenaka, J. Kikuchi, and T. Doke, OHZORA high energy particle observations, J. Geomag. Geoelectr., 37, 329-345, 1985.

Nishino, M., Y. Tanaka, T. Oguti, H. Yamagishi, and J. A. Holtet, Initial observation results with imaging riometer at Ny Alesund $(L=16)$, Proc. NIPR Symposium on Upper Atmosphere Physics, 6, 47-60, 1993. Nishino, M., K. Makita, K. Yumoto, F. Rodrigues, N. J. Schuch, and M. A. Abdu, Unusual ionospheric absorption characterizing energetic electron precipitation into the South Atlantic magnetic anomaly, Earth Planets Space, 54, 907-916, 2002.

Paulikas, G. A., Precipitation of particles at low and middle latitudes, Rev. Geophys. Space Phys., 3(5), 709-734, 1975.

Pinto, Jr. O. and W. D. Gonzalez, X ray measurements at the South Atlantic magnetic anomaly, J. Geophys. Res., 91(A6), 7072-7078, 1986.

Pinto, O. and W. D. Gonzalez, Energetic electron precipitation at the South Atlantic Magnetic Anomaly: review, J. Atmos. Terr. Phys., 5, 351-365, 1989.

Pinto, Jr. O., W. D. Gonzalez, and N. M. Paes Leme, VLF disturbances at the South Atlantic magnetic anomaly following magnetic storms, Planet. Space Sci., 38(5), 633-636, 1990.

Raben, V. J., D. E. Evans, H. H. Sauer, S. R. Sahm, and M. Huynh, Tiros/NOAA satellite space environment monitor data archive documentation: 1995 update, NOAA Tech. Memo. ERL SEL-86, 1995.

Tanaka, T., Low-latitude ionospheric disturbances: Results for March 22, 1979, and their general characteristics, Geophys. Res. Lett., 13(13), 1399-1402, 1986.

Torr, D. G., R. Martsha, R. Torr, C. G. Walker, and R. A. Hoffman, Particle precipitation in the South Atlantic geomagnetic anomaly, Planet. Space Sci., 23, 15-26, 1975.

Walt, M. and H. D. Voss, Proton precipitation during magnetic storms in August through November 1998, J. Geophys. Res., 109, A02201, doi:0.1029/2003JA010083, 2004.

M. Nishino (e-mail: stel_nishino@yahoo.co.jp), K. Makita, K. Yumoto, Y. Miyoshi, N. J. Schuch, and M. A. Abdu 